\title{
Mapping the sex determination locus in the hāpuku (Polyprion oxygeneios) using ddRAD sequencing
}

Jeremy K. Brown ${ }^{1 \dagger}$, John B. Taggart ${ }^{1 \dagger}$, Michaël Bekaert $^{1}$, Stefanie Wehner ${ }^{1}$, Christos Palaiokostas ${ }^{2}$, Alvin N. Setiawan ${ }^{3}$, Jane E. Symonds ${ }^{3,4}$ and David J. Penman ${ }^{1 *}$

\begin{abstract}
Background: Hāpuku (Polyprion oxygeneios) is a member of the wreckfish family (Polyprionidae) and is highly regarded as a food fish. Although adults grow relatively slowly, juveniles exhibit low feed conversion ratios and can reach market size in 1-2 years, making $P$. oxygeneios a strong candidate for aquaculture. However, they can take over 5 years to reach sexual maturity in captivity and are not externally sexually dimorphic, complicating many aspects of broodstock management. Understanding the sex determination system of $P$. oxygeneios and developing accurate assays to assign genetic sex will contribute significantly towards its full-scale commercialisation.

Results: DNA from parents and sexed offspring ( $n=57)$ from a single family of captive bred $P$. oxygeneios was used as a template for double digestion Restriction-site Associated DNA (ddRAD) sequencing. Two libraries were constructed using Sbfl - Sphl and Sbfl - Ncol restriction enzyme combinations, respectively. Two runs on an Illumina Miseq platform generated 70,266,464 raw reads, identifying 19,669 RAD loci. A combined sex linkage map (1367 cM) was constructed based on 1575 Single Nucleotide Polymorphism (SNP) markers that resolved into 35 linkage groups. Sex-specific linkage maps were of similar size (1132 and 1168 cM for male and female maps respectively). A single major sex-determining locus, found to be heterogametic in males, was mapped to linkage group 14. Several markers were found to be in strong linkage disequilibrium with the sex-determining locus. Allele-specific PCR assays were developed for two of these markers, Sphl6331 and Sphl8298, and demonstrated to accurately differentiate sex in progeny within the same pedigree. Comparative genomic analyses indicated that many of the linkage groups within the $P$. oxygeneios map share a relatively high degree of homology with those published for the European seabass (Dicentrarchus labrax).
\end{abstract}

Conclusion: $P$. oxygeneios has an XX/XY sex determination system. Evaluation of allele-specific PCR assays, based on the two SNP markers most closely associated with phenotypic sex, indicates that a simple molecular assay for sexing P. oxygeneios should be readily attainable. The high degree of synteny observed with D. labrax should aid further molecular genetic study and exploitation of hāpuku as a food fish.

Keywords: Hapuku, Sex chromosome, Mariculture, RAD-seq, New Zealand groper

\footnotetext{
* Correspondence: d.jpenman@stir.ac.uk

Jeremy K. Brown and John B. Taggart are joint first authors.

${ }^{\dagger}$ Equal contributors

'Institute of Aquaculture, School of Natural Sciences, University of Stirling,

Stirling FK9 4LA, Scotland, UK

Full list of author information is available at the end of the article
} 


\section{Background}

The hāpuku (Polyprion oxygeneios), or New Zealand groper, is a member of the wreckfish family (Polyprionidae). These large predatory fish range throughout the southern hemisphere and have been recorded in waters around New Zealand, southern Australia, southern America and a number of southern ocean archipelagos [1]. Although highly valued as a food and game fish, wild $P$. oxygeneios fisheries are relatively small scale and data on their exploitation status is limited [2-4]. P. oxygeneios juveniles are initially pelagic, moving to $>100 \mathrm{~m}$ deep demersal habitats at around 3-4 years of age, where they can go on to reach $100 \mathrm{~kg}$ over an estimated 50-60 year lifespan [3, 4]. Although the species is generally slow growing, analysis of wild populations indicates that juveniles grow very rapidly during the first three years $[3,4]$. These data are consistent with growth studies in captive juveniles of the closely related species, Polyprion americanus [5]. Moreover, growth studies undertaken by New Zealand National Institute of Water and Atmospheric Research (NIWA) have shown that $P$. oxygeneios can reach $1 \mathrm{~kg}$ in 12 months and $3 \mathrm{~kg}$ in $21-$ 24 months, with commercially competitive feed conversion ratios (FCR) in the region of 1-2 [6, 7]. Market studies indicate that farmed portion size fish and fillets from larger fish are highly regarded in both local and international markets, making this species a strong candidate for aquaculture [7].

NIWA have an experimental captive breeding programme for wild-caught $P$. oxygeneios broodstock $[8,9]$ but maintaining a reliable supply of high quality fingerlings remains an obstacle to commercialisation of the species [7]. Wild P. oxygeneios are communal spawners and readily breed in captivity without the need for complex environmental or physiological interventions $[8,9]$. However, their progeny take a relatively long time to reach sexual maturity and are not externally sexually dimorphic. Wild fish become sexually mature at an estimated 7-13 years, based on Western Australian [4] and New Zealand [3] stocks. Sexual maturation appears to occur slightly earlier in captive bred $P$. oxygeneios, such that male and female F1 stock can be differentiated at around 5 years of age using ultrasound imaging or evaluation of plasma vitellogenin or sex steroid levels [10]. All three of these methods only work reliably for adult captive broodstock in the run up to, and during the first half of, the August December spawning season [10]. It would be advantageous to be able to sex $P$. oxygeneios at a much earlier age for broodstock management.

Analysis of sex ratios and gonad morphology in wild stocks indicates that $P$. oxygeneios is a primary gonochorist [4], and more than 10 years of captive breeding studies have yet to produced evidence to the contrary [11]. Aside from the observation that sex ratios in captive families are usually balanced (NIWA, unpublished data), very little is known about sex determination in
P. oxygeneios. The mechanisms that determine sex in gonochoristic fish species are diverse; some rely purely on genetic determination, some on environmental cues and others on a combination of both [12, 13]. Although sex chromosomes are well differentiated in therian mammals and birds, which use male heterogamety $(\mathrm{XX} / \mathrm{XY})$ and female heterogamety (ZW/ZZ) to determine sex, respectively, the majority of fish species do not show sex chromosome differentiation. Sex chromosome pairs in fish species that do have $\mathrm{XX} / \mathrm{XY}$ or $\mathrm{ZW} / \mathrm{ZZ}$ sex-determining systems often show little or no heteromorphism [14], making sex identification problematic, even in species with known sex determination. Mapping the sex determination locus (or loci) using molecular markers would allow identification of sex-specific, or at least sex-linked, markers with which to develop molecular sexing methods.

The primary aim of the current study was to explore the genetics of sex-determination in hāpuku. Double digest Restriction-site Associated DNA (ddRAD) sequencing [15] was used to simultaneously identify and genotype a large number of novel Single Nucleotide Polymorphism (SNP) markers in the largest available captive-bred full-sib family for which phenotypic sex had been scored. The resultant genotypic data were used to construct the first genetic linkage maps for $P$. oxygeneios, and to search for potential sex-determining regions in this species. Two SNPs closely associated with phenotypic sex in the family were then developed as specific PCR assays and validated for their ability to differentiate genotypic sex. Additionally, the mapped ddRAD sequences were used to search for homologies with other 'model' fish species using comparative analyses.

\section{Methods}

\section{Sample collection}

All animal manipulations and handling were approved by the NIWA Animal Ethics Committee, in accordance with the national guidelines under the Animal Welfare Act 1999 of New Zealand. Wild-caught P. oxygeneios broodstock and F1 offspring were maintained at the NIWA aquaculture facility, Bream Bay, New Zealand [8]. Seven female and eight male broodstock were maintained in a $70 \mathrm{~m}^{3}$ communal spawning tank [9]. F1 juveniles from this batch spawning were fin clipped and tagged intraperitoneally with passive integrated transponders (PIT) [6]. Pedigrees were assigned using a proprietary pedigree analysis programme developed by AgResearch (New Zealand) whereby DNA profiles of the progeny were compared against all combinations of parents within the spawning tank [8]. F1 offspring from the largest represented full-sib family were sacrificed 432-439 days post-hatching (mean weight of $1454 \pm 116 \mathrm{~g}$ ) in November/December 2014. Gonads were fixed in $10 \%$ neutral buffered formalin and 
routinely processed, embedded in paraffin wax, sectioned at $5 \mu \mathrm{m}$ and stained with haematoxylin and eosin by New Zealand Veterinary Pathology [http://www.nzvp.co.nz]. F1 offspring were assigned phenotypic sex by histological examination of the gonads (Additional file 1: Figure S1). Fin clips from a single family (76 progeny; 38 males and 38 females, and two assigned parents) and 29 additional broodstock (14 males and 15 females) were collected into $100 \%$ ethanol and shipped dehydrated at ambient temperature to the Institute of Aquaculture, University of Stirling, UK.

\section{Preparation of genomic DNA}

After shipment fin clips were stored at $4{ }^{\circ} \mathrm{C}$ in $100 \%$ ethanol prior to use. For DNA extraction fin clips were blotted dry and 10-15 $\mathrm{mm}^{2}$ samples transferred into nuclease-free $1.7 \mathrm{~mL}$ microfuge tubes. Samples were then incubated for $2 \mathrm{~h}$ at $55{ }^{\circ} \mathrm{C}$ with end-over-end mixing in $200 \mu \mathrm{l}$ of CTAB buffer: $100 \mathrm{mM}$ pH 8.0 tris $\mathrm{HCl}$, $20 \mathrm{mM}$ EDTA, 1.4 M NaCl, $55 \mathrm{mM}$ hexadecyltrimethylammonium bromide, $0.2 \%$ 2-mercaptoethanol (SigmaAldrich, Gillingham, UK); and $5 \mu \mathrm{L}$ of proteinase $\mathrm{K}$ (10 $\mathrm{mg} / \mathrm{mL}$; Sigma-Aldrich). Once cooled to room temperature, $5 \mu \mathrm{L}$ of Rnase A ( $2 \mathrm{mg} / \mathrm{mL}$; Sigma-Aldrich) was added, the mixture then being incubated for a further $60 \mathrm{~min}$ at $37{ }^{\circ} \mathrm{C}$ with end-over-end mixing. Under a fume hood, samples were mixed well for $2 \mathrm{~min}$ with $110 \mu \mathrm{L}$ of chloroform (Sigma-Aldrich) before centrifugation for $3 \mathrm{~min}$ at $12,000 \mathrm{~g}$. Under a fume hood, $110 \mu \mathrm{L}$ of the upper aqueous phase was transferred into a fresh nuclease-free $1.7 \mathrm{~mL}$ microfuge tube, to which $8.3 \mu \mathrm{L}$ of $3 \mathrm{M}$ sodium acetate $\mathrm{pH} 5.4$ (Sigma-Aldrich) and $83 \mu \mathrm{L}$ of isopropanol (Sigma-Aldrich) were added. DNA was precipitated by repeated sharp inversion of the samples followed by centrifugation at $12,000 \mathrm{~g}$ for $1 \mathrm{~min}$. The supernatant was discarded and the pellet incubated in $1 \mathrm{~mL}$ of $70 \%$ aqueous ethanol overnight at ambient temperature with end-over-end mixing. Samples were centrifuged at $21,000 \mathrm{~g}$ for $5 \mathrm{~min}$ and the supernatant discarded, the pellet was then air dried for $5 \mathrm{~min}$ at $55^{\circ} \mathrm{C}$ and dissolved in $20 \mu \mathrm{L}$ of $5 \mathrm{mM}$ Tris $\mathrm{HCl}(\mathrm{pH}$ 8) overnight at $4{ }^{\circ} \mathrm{C}$. Total nucleic acid content and quality $(260 \mathrm{~nm} /$ $230 \mathrm{~nm}$ and $260 \mathrm{~nm} / 280 \mathrm{~nm}$ ratios) were determined by spectrometry (Nanodrop; Thermo Scientific, Hemel Hempstead, UK) before adjusting nucleic acid concentrations to $100 \mathrm{ng} / \mu \mathrm{L}$ with $5 \mathrm{mM}$ TrisHCl (pH 8). Double stranded DNA concentrations were then more accurately measured using a Qubit dsDNA Broad Range Assay Kit and Qubit Fluorometer (Invitrogen, Paisley, UK) and adjusted to $8 \mathrm{ng} / \mu \mathrm{L}$ with $5 \mathrm{mM}$ TrisHCl ( $\mathrm{pH} 8)$. As a final quality check $24 \mathrm{ng}$ of each sample was then separated by electrophoresis, with $50 \mathrm{ng}$ of Hind III digested lambda phage DNA as a size standard, in a $0.8 \%$ agarose gel, $0.5 \times$ TAE buffer $(20 \mathrm{mM}$ Tris, $10 \mathrm{mM}$ acetic acid, and $0.5 \mathrm{mM}$ EDTA $\mathrm{pH}$ 8.0) containing $100 \mathrm{ng} / \mathrm{mL}$ of ethidium bromide (EtBr) to allow appraisal of genomic DNA quality and quantity prior to ddRAD library preparation. Genomic DNA of sufficiently high quality for generating ddRAD libraries was obtained from both parents and 58 of the 76 offspring supplied. Of the remaining 18 offspring, genomic DNA obtained from 16 individuals was deemed to be too degraded for inclusion in the libraries but adequate for use in subsequent PCR assays.

\section{ddRAD library preparation and sequencing}

Following a modified version of the protocol described by Peterson et al. [15], two ddRAD libraries were prepared using different pairs of high fidelity restriction enzymes (REs) (New England Biolabs, UK; NEB); one using Sbfl (specific for the CCTGCA|GG motif) and SphI (specific for the GCATG|C motif) and the other using Sbfl and NcoI (specific for the C|CATGG motif). The libraries were prepared consecutively, two weeks apart. For each library, samples from offspring $(n=58)$ were prepared in duplicate, while parental samples $(n=2)$ were performed in sextuplicate, to provide a three-fold overrepresentation of parental sequences. For each replicate, $3 \mu \mathrm{L}$ of genomic DNA (24 ng) were individually digested at $37{ }^{\circ} \mathrm{C}$ for $45 \mathrm{~min}$ with $0.3 \mathrm{U}$ of each $\mathrm{RE}$ pair in a final volume of $6 \mu \mathrm{L} 1 \mathrm{x}$ CutSmart buffer (New England Biolabs). Samples were returned to ambient temperature for $5 \mathrm{~min}$, and then incubated for $10 \mathrm{~min}$, at ambient temperature, with $3 \mu \mathrm{L}$ of unique combinations of RAD-specific P1 (6 nM) and P2 (72 nM) pairedend adapters [16] that included 5 or 7 bp barcodes (Additional file 2: Table S1). Barcoded adapters were designed such that adapter-genomic DNA combinations did not reconstitute RE sites, while residual RE activity limited concatemerisation of genomic fragments. The individual reactions were then incubated for $2 \mathrm{~h}$ at ambient temperature with $3 \mu \mathrm{L}$ of ligase mastermix (1 mM rATP (Promega, Mannheim, Germany) and 2000 cohesive-end Units per $\mu \mathrm{g}$ of DNA of T4Ligase (New England Biolabs) in $1 \times$ CutSmart buffer). Ligase activity in each individual reaction was stopped by the addition of $30 \mu \mathrm{L}$ PB buffer ( $\mathrm{pH}$ indicator added) from a MinElute PCR purification kit (Qiagen, Manchester, UK) and the individual samples then pooled into one tube. The pooled reaction was $\mathrm{pH}$ adjusted with c. $2 \mu \mathrm{L} 3 \mathrm{M}$ sodium acetate $\mathrm{pH} 5.4$ and sequentially loaded, in $550 \mu \mathrm{L}$ aliquots, onto a single Minelute spin column and processed as per manufacturer's instructions. The pooled library was eluted from the column in $2 \times$ $36 \mu \mathrm{L}$ warmed EB buffer $\left(60^{\circ} \mathrm{C}\right)$ and stored on ice.

Size selection was performed by chilled electrophoresis in an EtBr-free $1.2 \%$ agarose gel, 0.5× TAE buffer, loading the precooled sample (c. $68 \mu \mathrm{L}$ of adapter ligated 
DNA mixed with $10 \mu \mathrm{L}$ of $6 \times$ Ficol-based gel loading buffer into a single $25 \mathrm{~mm}$ wide lane flanked by lanes loaded with 710/416 base pair (bp) size markers. The gel was run at constant voltages of $45 \mathrm{~V}$ for $3 \mathrm{~min}, 60 \mathrm{~V}$ for $3 \mathrm{~min}$ and $90 \mathrm{~V}$ for around $70 \mathrm{~min}$ until the bromophenol blue dye front travelled approximately $3.5 \mathrm{~cm}$ from the origin. The central $2.2 \mathrm{~cm}$ wide by $4 \mathrm{~cm}$ long portion of the gel, that included the desired sample fractions (co-migrating roughly with the bromophenol blue marker dye), was excised with a scalpel and stored at $4{ }^{\circ} \mathrm{C}$ while the outer portion of the gel was post stained $(0.5 \mu \mathrm{g} / \mathrm{mL} \mathrm{EtBr}$ in $0.5 \times \mathrm{TAE}$ buffer $)$ for $10 \mathrm{~min}$. Under UV transillumination, notches were cut in the outer portion of the gel to mark the location of the 710/416 bp size markers. The outer portion of the gel was washed in nuclease-free water and the central portion of the gel, containing the sample, replaced. Using the notches as guides, a horizontal slice was excised from the central section of the gel; $\sim 6 \mathrm{~mm}$ thick, corresponding to a region containing c. 416 bp to c. 710 bp DNA fragments. The excised gel slice was processed through a spin-column (MinElute Gel Clean Up kit; Qiagen). The gel was dissolved in 3 volumes QG buffer at ambient temperature for $30 \mathrm{~min}$ with end-over-end mixing, the entire solution being sequentially loaded onto a single Minelute column and further processed following the manufacturer's recommended protocol. The library template DNA was eluted from the column in two $35 \mu \mathrm{L}$ volumes of warmed $\mathrm{EB}$ buffer $\left(60{ }^{\circ} \mathrm{C}\right.$ ) and stored on ice (final volume $\sim 65 \mu \mathrm{L}$ ).

Following optimisation of PCR conditions, to identify the minimum number of PCR cycles required to produce sufficient product for sequencing, a bulk amplification of each library $(400 \mu \mathrm{L})$ was undertaken. For both libraries this large scale PCR comprised $32 \mu \mathrm{L}$ library template, $9.6 \mu \mathrm{L}$ combined $10 \mu \mathrm{M}$ Illumina compatible P1 and P2 adapter specific primers [16], $200 \mu \mathrm{l}$ Q5 Hot Start HF 2x Master Mix (New England Biolabs) and $160 \mu \mathrm{L}$ nuclease-free water. After thorough mixing of the components, this mastermix was split into 32 times $12.5 \mu \mathrm{L}$ separate reactions for PCR. Cycling conditions were: $98{ }^{\circ} \mathrm{C}$ for $1 \mathrm{~min}$; then 12 cycles of $98{ }^{\circ} \mathrm{C}$ for $10 \mathrm{~s}$, $65{ }^{\circ} \mathrm{C}$ for $15 \mathrm{~s}$ and $72{ }^{\circ} \mathrm{C}$ for $40 \mathrm{~s}$; a final $72{ }^{\circ} \mathrm{C}$ for $3 \mathrm{~min}$. Following PCR the 32 aliquots were recombined. To confirm successful amplification, $5 \mu \mathrm{L}$ of the reaction was checked by electrophoresis $(1.5 \%$ agarose/TAE buffer gel; $100 \mathrm{ng} / \mathrm{mL}$ EtBr). The remainder was mixed with 3 volumes PB buffer, acidified with $2.5 \mu \mathrm{L}$ of $3 \mathrm{M}$ sodium acetate, and loaded in $550 \mu \mathrm{L}$ aliquots onto a single MinElute PCR purification spin-column. The column was processed as per manufacturer's instructions, the library being finally eluted in two $28 \mu \mathrm{L}$ volumes of warmed EB buffer $\left(60^{\circ} \mathrm{C}\right)$. The library was further cleaned, to maximise removal of small fragments (< c. $200 \mathrm{bp}$ ), using an equal volume of Agencourt AMPure XP paramagnetic beads (Beckman Coulter, High Wycombe, UK) following the manufacturer's instructions. The purified library was finally eluted in $18 \mu \mathrm{L}$ EB buffer. For QC purposes $1 \mu \mathrm{L}$ of purified library was visualised and sized by electrophoresis $(1.5 \%$ agarose gel; $0.5 \times$ TAE buffer; $100 \mathrm{ng} / \mathrm{mL}$ $\mathrm{EtBr})$. DNA concentrations of both the purified library and the PCR template were measured by fluorimetry (Qubit dsDNA high sensitivity Assay Kit, Life Technologies, Paisley, UK). Finally, by taking into account the library size range and proportion of template present in the bulk PCR reaction, the purified library was diluted to $10 \mathrm{nM}$ (amplicon equivalent) in EB buffer, $0.1 \%$ Tween20, and stored at $-20{ }^{\circ} \mathrm{C}$ until sequenced.

Sequencing was performed on the Illumina MiSeq platform (162 base paired end reads using a 300 base, v2 chemistry kit; Illumina, Cambridge, UK). Raw reads were processed using RTA 1.18.54 (Illumina). The raw sequence data from this study have been submitted to the EBI Sequence Read Archive (SRA) study PRJEB11817.

\section{Genotyping ddRAD alleles}

The MiSeq generated reads were processed using a software pipeline designed specifically for RAD analysis, Stacks version 1.27 [17]. First, the process_radtags function was used to demultiplex the individual samples. During this step sequence reads with quality scores below 20, missing either restriction site or with ambiguous barcodes were discarded. Barcodes were removed and all sequences trimmed to be 135 bases long. For the purposes of this analysis paired-end reads were treated as separate loci, Read 2 sequences being appended to Read 1 sequence files. These sequences were assigned to RAD loci and genotypes using the 'denovo_map.pl' component of Stacks. The key parameter values employed in identifying RAD loci were; a minimum stack depth $(\mathrm{m})$ of 10 , a maximum of 2 mismatches allowed in a locus (M) in an individual and up to 1 mismatch between loci when building the catalog (n).

\section{Genetic linkage map construction}

Taking a conservative approach, only data from RAD loci identified as containing one or two SNPs, i.e., those most likely to reflect true polymorphisms, were considered for analysis. These SNPs were further filtered, including only those identified in both parents and $\geq 75 \%$ of offspring for analysis, and mapped using Lep-Map 2 [18]. Phenotypic sex was incorporated into the data set in both XX/XY and ZW/ZZ formats. Markers deviating from expected Mendelian segregation $(P<0.01)$ were excluded from further analysis using the option "dataTolerance = 0.01" within the Filtering module. Lep-Map 2 was used to generate male, female and consensus maps during the execution of all subsequent modules by including the commands; "informativeMask $=13$ " for the male map, 
"informativeMask = 23" for the female map and "informativeMask $=123$ " for the consensus map. Linkage groups were generated using the modules SeparateChromosomes and JoinSingles with options "sizeLimit $=10$ " and "lodLimit $=8$ " to limit linkage group generated to $\geq 10$ markers with a minimum logarithm of the odds (LOD) value of 8 . The module "OrderMarkers" with options "useKosambi $=1$ " and "maxDistance $=50$ " was used to determine the order and distance between markers in centiMorgans (cM) using the Kosambi mapping function [19], splitting linkage groups that had gaps of $\geq 50 \mathrm{cM}$ between markers. The option "sexAveraged = 1 " was included during execution of OrderMarkers when constructing the consensus map. Similar parameters were also used to construct a consensus map using the R/OneMap package [20] release 2.0-4. The genetic maps were drawn and aligned using Genetic-Mapper v0.6 [21].

\section{Association analysis}

Association analysis was performed using the R/SNPassoc package [22] release 1.9-2 to test for associations between SNP genotypes and phenotypic sex using the function WGassociation with options "model = "codominant". Bonferroni correction was used to counteract the problem of multiple comparisons when determining the significance of observed results.

\section{Allele-specific PCR assays}

Allele-specific PCR primers [23] were designed for the two SNPs that were most closely associated with phenotypic sex using Primer Select software (DNASTAR Inc. Madison, USA). Specificity was achieved by designing two forward primers, one for each allele, with the SNP at the 3' end preceded by a mismatched nucleotide; and a single common reverse primer. Both assays performed optimally with a $60{ }^{\circ} \mathrm{C}$ annealing step. Allele-specific PCR was performed on a subsample of RAD-screened offspring (for assay validation: $n=10$ ) and on other offspring from the same pedigree $(n=16)$, which were not included in the ddRAD libraries due to their relatively poor genomic DNA integrity. Allele-specific PCR was also performed on samples from unrelated stock to estimate SNP allelic frequencies and association with phenotypic sex in the wider population $(n=29)$. Amplicons were separated by electrophoresis ( $2 \%$ agarose gel, $0.5 \times$ TAE buffer, containing $100 \mathrm{ng} / \mathrm{mL}$ ethidium bromide) with repeat loading of pairs of allele-specific amplicons at 5 min intervals into the same wells, to resolve double-banded phenotypes for heterozygous and single-banded phenotypes of different mobility for both homozygous phenotypes.

\section{Comparative genomics}

Consensus marker sequences were used as a query for homology searches against available fish genomes with
BLASTn v.2.2.28+ [24]. Reference sequences of Astyanax mexicanus (AstMex102), Danio rerio (GRCz10), Dicentrarchus labrax (dicLab1), Gadus morhua (gadMor1), Gasterosteus aculeatus (BROAD_S1), Latimeria chalumnae (LatCha1), Lepisosteus oculatus (LepOcu1), Oreochromis niloticus (Orenil1.1), Oryzias latipes (HdrR), Petromyzon marinus (Pmarinus_7.0), Poecilia formosa (Poecilia_formosa-5.1.2), Salmo salar (ICSASG_v2), Takifugu rubripes (FUGU 4.0), Tetraodon nigroviridis (TETRAODON8.0) and Xiphophorus maculatus (Xipmac4.4.2) were downloaded from the NCBI Genome Assembly database.

For the further analyses only RAD loci present in the $P$. oxygeneios linkage map and their putative homologs in the other fish genomes were taken into account. These RAD loci were repeat masked and the best hit for each RAD locus was deemed to be homologous if it covered at least $50 \%$ of the sequence with at least $50 \%$ identity. Detected homologies within D. labrax were visualized with Circos [25] release 0.68.

\section{Results \\ ddRAD sequencing}

Total numbers of raw reads and filtered reads obtained from each library using the Illumina MiSeq platform are shown in Fig. 1. Overall the performance and yield of both RAD loci and informative SNPs was broadly similar for the two libraries. Consistent with their deliberate over-representation in both libraries, in order to more robustly identify true SNPs, 2 to 3 fold more reads were obtained for the parents (Additional file 2: Table S1). After removal of low quality sequences and reads with missing or ambiguous barcodes, $86.6 \%$ of reads in the Sbfl - SphI library and $85.5 \%$ of reads in the Sbfl - NcoI library were retained. One of the offspring, \#86, was approximately 10 -fold underrepresented $\mathrm{cf}$. the others in both libraries and was excluded from subsequent analysis on this basis (Additional file 2: Table S1). De novo building and genotyping of loci with Stacks identified a total of more than 10,000 RAD loci in each library. Of these, 1360 in the Sbfl - SphI library and 1598 in the Sbfl - Ncol library contained 1-2 SNPs. A combined total of 1667 markers were informative in one or both sexes, present in at least $75 \%$ of the offspring and adhered to expected Mendelian segregation ratios $\left(\chi^{2}\right.$ $p$-value $>0.01)$. All of these markers were subsequently used to construct genetic linkage maps (Additional file 3: Table S2). The 1609 bi-allelic markers present in this panel were also used in association analysis.

\section{Genetic linkage maps}

Consensus, female and male genetic linkage groups were constructed for $P$. oxygeneios using Lep-Map 2 (Table 1). The consensus map was constructed using 1667 informative markers, 1575 of which mapped to 35 linkage 


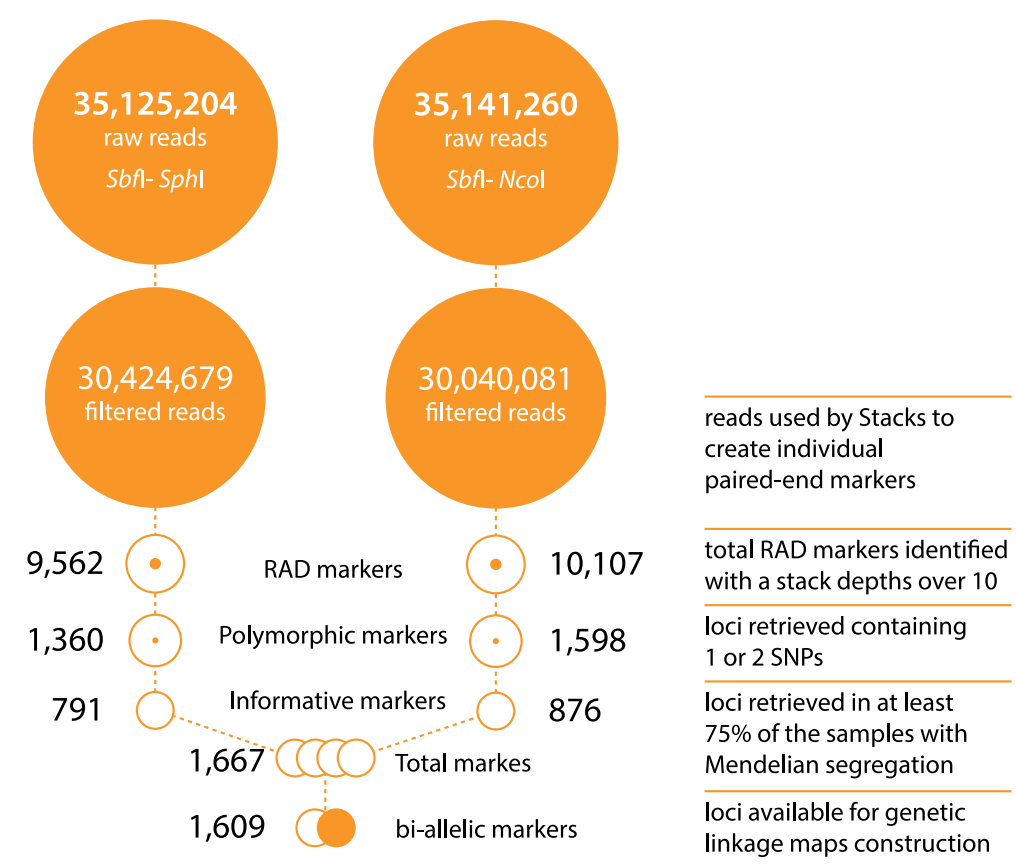

Fig. 1 Pictorial summary of the sequencing pipeline. Total numbers of reads obtained from each library are shown; raw and filtered for low quality sequences (quality score $<20$ ) and reads with missing or ambiguous barcodes, followed by the number of polymorphic ddRAD markers identified by Stacks, and the number of informative markers that were subsequently used to generate genetic linkage maps and in genome-wide association studies

groups with $\geq 10$ markers, spanning a total distance of 1366.4 cM (Fig. 2, Additional file 4: Table S3). Similar results were obtained using R/OneMap (data not shown). The $1168 \mathrm{cM}$ female-specific map was constructed using 819 markers that were informative in the female (Additional file 5: Figure S2, Additional file 6: Table S4). Similarly, the $1132 \mathrm{cM}$ male-specific map was constructed using 878 markers that were informative in the male (Additional file 7: Figure S3). In both cases, linkage group identities were matched to those of the consensus map (Fig. 3, Additional file 8: Table S5). When phenotypic sex of the offspring was coded as $\mathrm{XX} / \mathrm{XY}$ genotypes and included in the linkage analysis, 'sex' localised to the end of linkage group 14 in both the consensus and male maps but was absent from the female-specific map (Fig. 3). When scored as ZW/WW genotypes, 'sex' remained unlinked in all three map analyses.

\section{Association analysis}

Association analysis identified seven markers that appear to be significantly associated with phenotypic sex in $P$. oxygeneios (Fig. 4). When ordered using the P. oxygeneios consensus linkage map, all seven markers located to one end of linkage group 14, adjacent to male phenotypic sex (Fig. 4b). The two markers most closely associated with male phenotypic sex in linkage group 14 (Fig. 3: SphI6331 and SphI8298) also showed the closest association with phenotypic sex association analysis (Fig. 4b: SphI6331 $P=1.4 \times 10^{-15}$ and SphI8298 $\left.P=4.5 \times 10^{-13}\right)$.

\section{Sex prediction using allele-specific PCR assays}

Allele-specific PCR primers were designed for markers SphI6331 and SphI8298 (Additional file 9: Table S6). DNA from known RAD-genotyped samples (5 males and 5 female offspring), together with DNA from 16 other phenotypically sexed offspring were screened, i.e., those samples for which extracted DNA was considered to be too degraded for inclusion in the ddRAD libraries. For SphI6331, the sire was heterozygous (AT) and the dam was homozygous (TT); 25 progeny conformed to this pattern, while one female was heterozygous (Additional file 10: Figure S4). For SphI8298, the sire was heterozygous (CT) and the dam was homozygous (TT): all male progeny were TT and all female progeny were CT (Additional file 10: Figure S4). The A allele of SphI6331 thus appears to be associated with the $\mathrm{Y}$ sex-determining allele in this family, while for SphI8298 the paternal T allele appears to be associated with the $\mathrm{Y}$ (and the paternal $\mathrm{C}$ allele with the $\mathrm{X}$ ). For the addition 29 unrelated broodstock, observed heterozygosity was relatively high at both loci (SphI6331 $=0.38$ and SphI8298 = 0.34) but phenotypic sex within this group was not associated with genotype at either locus. 
Table $1 P$. oxygeneios linkage maps

\begin{tabular}{|c|c|c|c|c|c|c|}
\hline \multirow{2}{*}{$\begin{array}{l}\text { Linkage } \\
\text { group }\end{array}$} & \multicolumn{2}{|c|}{ Consensus } & \multicolumn{2}{|l|}{ Female } & \multicolumn{2}{|l|}{ Male } \\
\hline & Markers & Size $(c M)$ & Markers & Size (cM) & Markers & Size (cM) \\
\hline 1 & 90 & 58.30 & 63 & 54.7 & 44 & 60.39 \\
\hline 2 & 78 & 44.24 & 41 & 49.55 & 50 & 21.08 \\
\hline 3 & 75 & 57.53 & 46 & 32.34 & 39 & 97.38 \\
\hline 4 & 73 & 50.80 & 39 & 65.7 & 49 & 48.58 \\
\hline 5 & 75 & 50.35 & 43 & 46.14 & 35 & 21.09 \\
\hline 6 & 70 & 53.60 & 37 & 46.13 & 47 & 87.47 \\
\hline 7 & 65 & 52.49 & 48 & 88.22 & 27 & 34.15 \\
\hline 8 & 65 & 59.89 & 40 & 60.08 & 45 & 62.07 \\
\hline 9 & 66 & 64.62 & 23 & 45.99 & 45 & 64.04 \\
\hline 10 & 69 & 55.08 & 28 & 42.49 & 44 & 40.56 \\
\hline 11 & 66 & 53.34 & 23 & 19.45 & 34 & 54.75 \\
\hline 12 & 58 & 51.29 & 37 & 50.72 & 28 & 46.94 \\
\hline 13 & 57 & 36.26 & 25 & 56.26 & 43 & 19.34 \\
\hline 14 & 62 & 70.77 & 27 & 57.21 & 33 & 62.17 \\
\hline 15 & 57 & 40.05 & 33 & 45.64 & 26 & 41 \\
\hline 16 & 55 & 29.51 & 22 & 26.68 & 33 & 19.32 \\
\hline 17 & 47 & 39.60 & 17 & 40.31 & 38 & 22.84 \\
\hline 18 & 47 & 41.52 & 12 & 43.12 & 33 & 31.64 \\
\hline 19 & 53 & 51.49 & 27 & 47.33 & 27 & 52.28 \\
\hline 20 & 42 & 46.55 & 13 & 32.59 & 37 & 35.53 \\
\hline 21 & 43 & 32.64 & 10 & 8.01 & 35 & 33.42 \\
\hline 22 & 30 & 39.69 & 10 & 26.42 & 17 & 26.43 \\
\hline 23 & 24 & 34.78 & 18 & 38.11 & 15 & 49.47 \\
\hline 24 & 23 & 33.99 & 23 & 28.25 & - & - \\
\hline 25 & 21 & 7.07 & 10 & 0 & 12 & 12.33 \\
\hline 26 & 19 & 14.19 & 17 & 10.55 & - & - \\
\hline 27 & 19 & 11.61 & 10 & 1.76 & 11 & 17.64 \\
\hline 28 & 19 & 37.49 & 16 & 31.47 & - & - \\
\hline 29 & 18 & 19.41 & - & - & 18 & 19.41 \\
\hline 30 & 23 & 25.18 & 15 & 4 & 13 & 50.55 \\
\hline 31 & 17 & 37.55 & 17 & 21.15 & - & - \\
\hline 32 & 13 & 12.62 & 13 & 12.62 & - & - \\
\hline 33 & 13 & 12.64 & - & - & - & - \\
\hline 34 & 13 & 36.74 & 10 & 33.18 & - & - \\
\hline 35 & 10 & 3.51 & 10 & 1.76 & - & - \\
\hline Total & 1575 & 1366.39 & 819 & 1167.93 & 878 & 1131.87 \\
\hline
\end{tabular}

\section{Comparative genomics}

Homology searches were performed against other fish genomes using the RAD loci included in the $P$. oxygeneios linkage map (Fig. 5, Additional file 11: Table S7). This was undertaken primarily in order to validate the map build but also to establish the extent to which existing fish genome resources could prove informative for future studies of this species.

The fewest 'homologous' loci were found in the comparison with $L$. oculatus and L. chalumnae, the only non-teleost species in the set of genomes explored $(P$. marinus were used as an outgoups in Fig. 5a and Table 2). Homologous sequences to more than $200 P$. oxygeneios ddRAD loci were found in O. niloticus, G. aculeatus, $X$. maculatus, $P$. formosa, $T$. rubripes, $O$. latipes and T. nigroviridis (Fig. 5a, Table 2). Of all the species examined, D. labrax exhibited by far the highest degree of homology with $P$. oxygeneios. Approximately $75 \%$ of the analysed sequences in $P$. oxygeneios have homologous sequences within the $D$. labrax genome (Fig. 5). P. oxygeneios linkage groups also appear to show a high degree of homology with $D$. labrax linkage groups (Fig. 5b), as every linkage group of $P$. oxygeneois is clearly linked to one linkage group in the D. labrax with exception of LG 14. Loci located in LG 14 of $P$. oxygeneois are mainly split between linkage groups 3 and 14 in D. labrax with marker order within each of these two D. labrax groups being highly conserved with P. . oxygeneois (Fig. 5c). Overall, there is a strong correlation of each hāpuku linkage group to a single LG not only in D. labrax but also in O. niloticus and G. aculeatus, where marker order also appears to be generally conserved (Additional file 11: Table S7). These observations support the contention that the hāpuku linkage map is reliable and robust.

\section{Discussion}

In the absence of pre-existing sequence or karyotype data, sequencing of two independent ddRAD libraries from a single full-sib family on an Illumina MiSeq has shown that $P$. oxygeneios has an $\mathrm{XX} / \mathrm{XY}$ sex determination system and allowed identification of several SNPs that are associated with phenotypic sex. In a departure from the methodology described by Peterson et al. [15], individual samples were pooled immediately after ligation with barcodes and prior to PCR amplification during preparation of both $P$. oxygeneios ddRAD libraries. This approach greatly reduces both the time required to prepare libraries and the potential for fragment size variation caused by size-selecting smaller groups of individuals prior to pooling into a single library for sequencing. The relative consistency in the number of reads obtained among individuals in both libraries (Additional file 2: Table S1) validates this approach. The use of two different RE pairs, which shared a common eight base recognition RE, Sbfl, but used different 6 base recognition REs SphI and Ncol to generate ddRAD fragments, resulted in the identification of approximately twice as many mappable RAD markers as either pair used in isolation. Only 43 

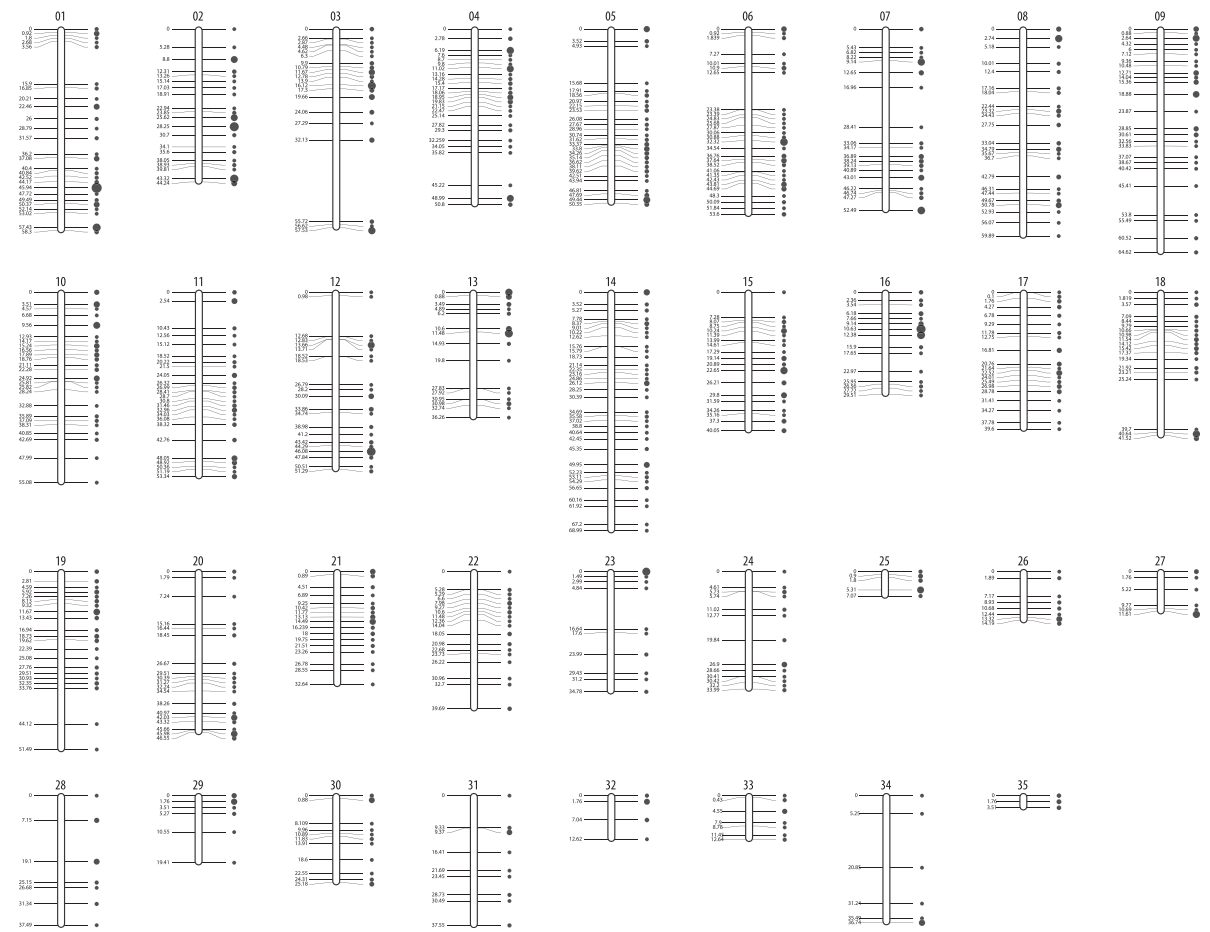

Fig. 2 P. oxygeneios Genetic linkage map. The consensus map generated using Lep-Map 2 software contained a total of 1575 informative SNP markers arranged into 35 linkage groups. The positions on the left side of the chromosomes are in centiMorgans. The diameters of the filled circles on the right hand side are proportionate to the number of markers at those positions. A more detailed map is provided in Additional file 3: Table S2

mapped loci were common to both libraries. In total, this approach produced 1667 SNPs that were informative for the purposes of genetic map construction, 1609 of which were bi-allelic and available for association analysis.
In the absence of karyotype information for $P$. oxygeneios, a relatively high minimum LOD score of 8 was used to construct the genetic linkage map, resulting in 35 linkage groups being identified. This is perhaps higher than the number of chromosomes that one might

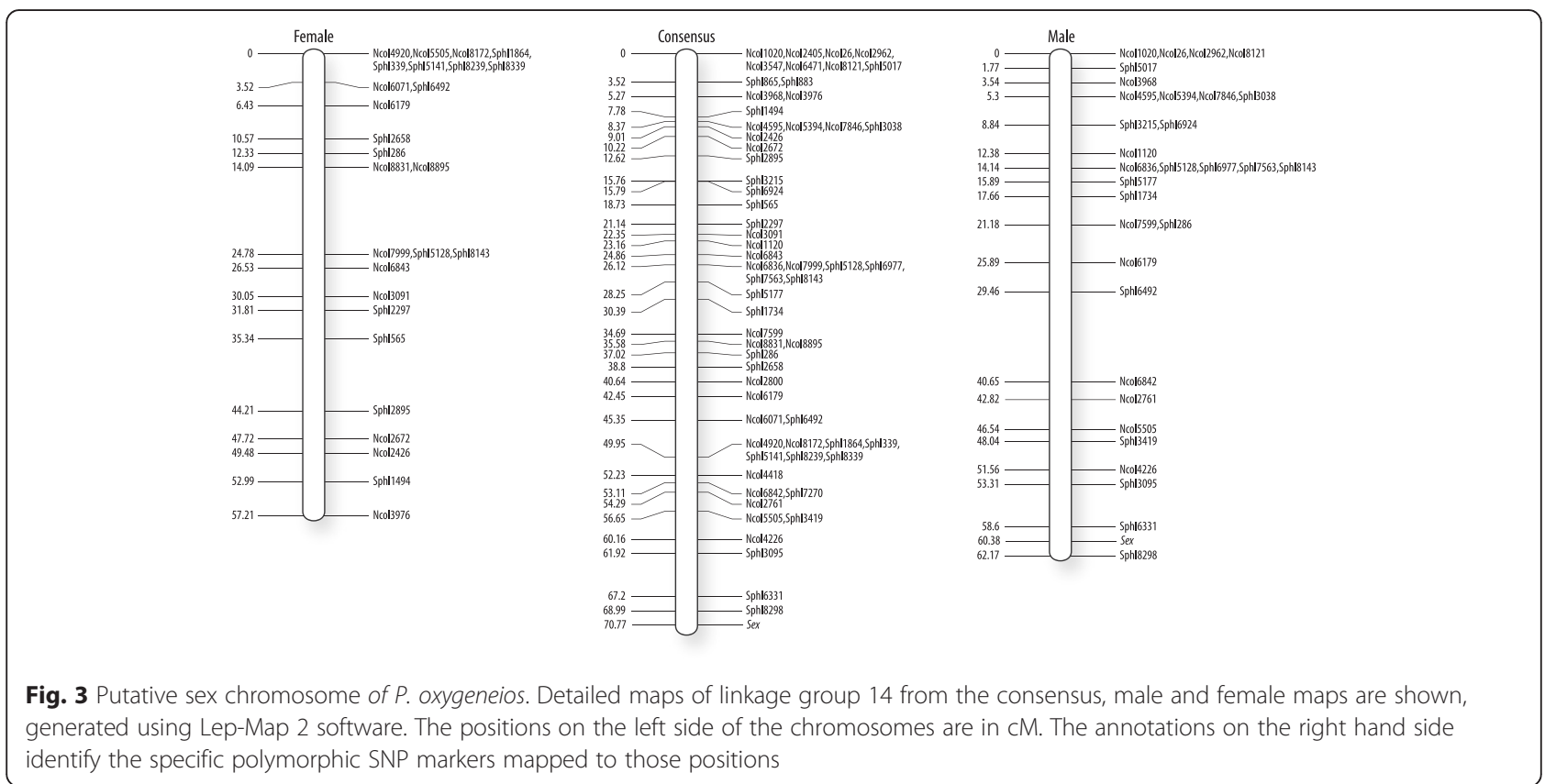




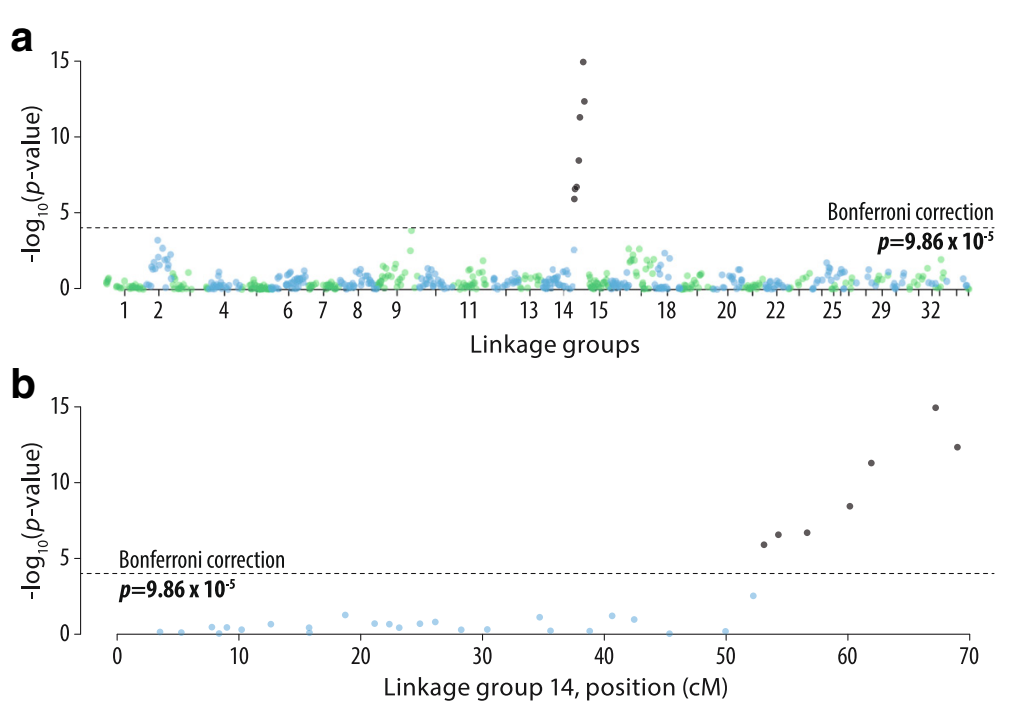

Fig. 4 Association analysis of informative SNPs and phenotypic sex. a A plot of the association of 543 unique positions (identified by 1522 informative SNPs) with phenotypic sex, scaled along the $x$-axis to their position (CM) in the P. oxygeneios genetic linkage map. $\mathbf{b}$ A higher resolution representation of the portion of linkage group 14 adjacent to the putative sex-determining locus, showing SNP marker identities. Dashed line represents Bonferroni corrected significance level $(P<0.05)$, based on 543 unique observations

expect to observe in $P$. oxygeneios, based on its relative homology with D. labrax, which has 24 pairs [26]. It was considered preferable to split linkage groups where there were large gaps between markers, rather than join potentially independent linkage groups. A drawback associated with this approach is that if phenotypic sex was not closely linked with any SNPs identified in the library then it might be excluded from the map completely. Fortunately, two of the markers with SNPs that were informative in the male offspring, SphI6331 and SphI8298, were located within $2 \mathrm{cM}$ of phenotypic sex on linkage group 14. The association analysis and subsequent allele-specific PCR screening of the offspring that were not included in the libraries strongly supported these associations. Taken collectively, these findings indicate that $P$. oxygeneios has an $\mathrm{XX} / \mathrm{XY}$ sex determination system, in which the major sex determining region is located in linkage group 14 and that SNPs in markers a

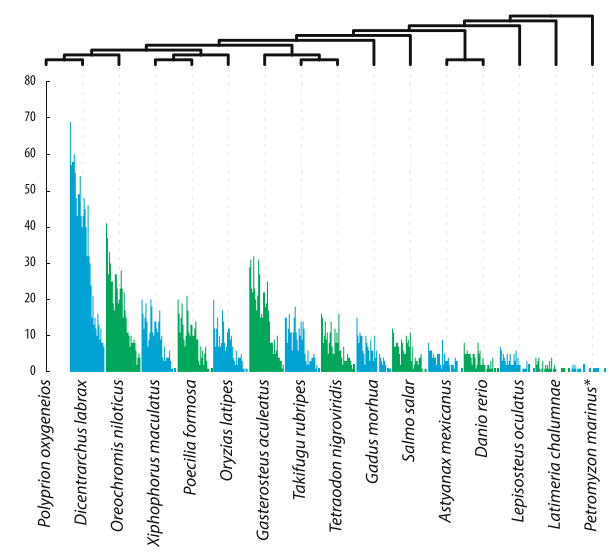

b

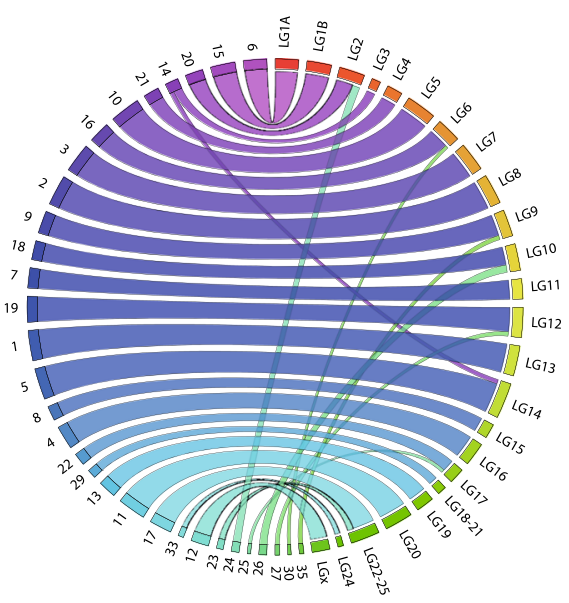

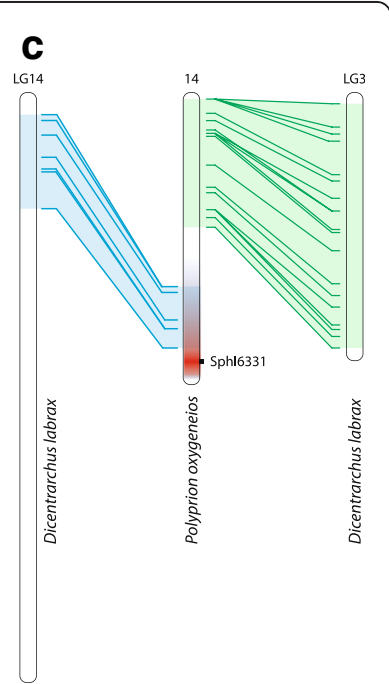

Fig. 5 Comparative genomics analysis. a The percentage of $P$. oxygeneios ddRAD sequences per linkage group that showed homology with sequences from other fish genomes, listed on the $x$-axis; ${ }^{*} P$. marinus: outgoup. The phylogenetic tree provided above the graph is based on recent classifications [30-32]. b Schematic of homology between proposed linkage groups (1-35) and published D. labrax linkage groups. Only blocks of 2 markers or more are illustrated; The complete comparative analysis for all species is summarised in Additional file 11: Table S7. c Graphic identifying syntenic regions between P. oxygeneios LG 14 and D. labrax linkage groups 
Table 2 Comparative genomics of $P$. oxygeneios

\begin{tabular}{lll}
\hline Species & Common name & $\begin{array}{l}\text { Number of } \\
\text { homologous loci }\end{array}$ \\
\hline Dicentrarchus labrax & European seabass & 1182 \\
Oreochromis niloticus & Nile tilapia & 616 \\
Gasterosteus aculeatus & Three-spined stickleback & 525 \\
Xiphophorus maculatus & Southern platyfish & 323 \\
Poecilia formosa & Amazon molly & 310 \\
Takifugu rubripes & Japanese puffer & 279 \\
Oryzias latipes & Medaka & 251 \\
Tetraodon nigroviridis & Green spotted puffer & 247 \\
Salmo salar & Atlantic salmon & 185 \\
Gadus morhua & Atlantic Cod & 180 \\
Astyanax mexicanus & Mexican tetra & 111 \\
Danio rerio & Zebrafish & 108 \\
Lepisosteus oculatus & Spotted gar & 84 \\
Latimeria chalumnae & Coelacanth & 39 \\
Petromyzon marinus & Lamprey & 17 \\
\hline
\end{tabular}

Number of mapped RAD loci in Hāpuku that shared homology with other fish genomes. ${ }^{\text {a }}$. marinus: outgoup

SphI6331 and SphI8298 are in strong linkage disequilibrium with the gene(s) within this locus.

Given the wide range of sex determination systems that operate in fish $[13,14]$, the discovery that sex determination in $P$. oxygeneios appears to be monogenic is encouraging with respect to developing sex determination assays. The results of the allele specific PCR assays for the two most closely associated loci, SphI6331 and SphI8298, appear to bear this out. However, the alleles at these loci are not sex-specific. This is illustrated by the fact that while the sire is heterozygous at SphI8298, the male offspring in the family were nearly all homozygous at this locus and thus the SphI8298 genotype would not be informative in the next generation. This is consistent with SphI8298 being located near the sex-determining locus, and likely to segregate with sex, but playing no direct role in sex determination. Although SphI6331 was heterozygous in both sire and male offspring it is located at around the same distance $(\sim 2 \mathrm{cM})$ from the sex determination locus as SphI8298. Additionally, the allelespecific PCR assay for SphI6331 was not $100 \%$ accurate at predicting sex, which is consistent with the ddRAD library results and probably reflects meiotic crossover in the $2 \mathrm{cM}$ interval between this marker and the sex determination locus. However, a "false" positive band (corresponding to A) in what was expected to be a homozygous TT female could also be an artefact of the allele-specific primers [23] initiating the PCR at the nucleotide immediately after the mismatch. This could account for the slightly smaller amplicon observed in this sample and the faint band at the same size observed in one of the other females. This may be resolved by further optimisation of the PCR conditions or adoption of potentially more stringent allele specific assays, such as Kompetitive Allele Specific PCR [27]. Given that heterozygous individuals appear to be relatively common in the wider population (0.38-0.41), it seems likely that these markers will be useful outside the current family where parental genotypic information is available and communal spawning groups can be structured to ensure that either, or both, markers are informative in the offspring. However, it may be necessary to extend the RAD sequencing studies to additional, larger, families or outbred populations to obtain the resolution required to identify markers that are more closely sex-linked than those identified in the current study. Ultimately, the genetic map generated in the current study will make possible mapping and association studies for any trait of interest with a view to long-term genetic improvement of captive $P$. oxygeneios lines [28].

Homology searches, comparing the RAD loci of $P$. oxygeneios with other fish genomes, demonstrate that a high degree of sequence homology exists with the European sea bass, D. labrax at chromosomal level, and to a lesser extent with $O$. niloticus and G. aculeatus. Marker order within linkage groups is also conserved, suggesting that the D. labrax genome, in particular, should be a valuable resource to aid future $P$. oxygeneios genetic studies. In contrast to what appears to be a relatively simply monogenic sex determination system in $P$. oxygeneios, sex determination in D. labrax is regulated through a combination of polygenic and environmental factors $[26,29]$. Interestingly, $P$. oxygeneios linkage group 14 is unusual in that its markers show sequence homology with sequences in multiple chromosomes in D. labrax.

\section{Conclusions}

The current study has produced the first-ever genetic linkage map of $P$. oxygeneios, using ddRAD sequencing. The map consists of 1575 loci in 35 linkage groups and supports the location of a single sex-determining region near one end of linkage group 14 in the male-specific and consensus maps. This is strongly indicative that $P$. oxygeneios has an XX/XY sex determination system. This study has also identified several novel markers that are in strong linkage disequilibrium with the gene(s) within the sex-determining region, albeit within the single family analysed. Allele-specific PCRs were developed for two of these markers, SphI6331 and SphI8298, and demonstrated to be able to accurately predict male and female offspring within the same family. Further analysis of unrelated individuals showed no population-wide association with phenotypic sex, but showed that heterozygosity is relatively common at both loci in the wider population, indicating that they are likely to be useful markers when sexing individuals within a pedigree 
situation. Finally, comparative genomic analyses indicate that many of the linkage groups within the $P$. oxygeneios map share a relatively high degree of homology with those published for D. labrax and that marker order within linkage groups appears highly conserved. It is hoped that these findings will lead to the development of more robust assays for genotypic sex, allowing a much greater degree of control of sex ratios in brood stock management, ongrowing and, ultimately, lead to genetic improvement of this promising new aquaculture species.

\section{Additional files}

Additional file 1: Figure S1. Phenotypic sexing. Representative histological presentation of female (A and B) and male (C and D) $P$. oxygeneios gonads at 432-439 days post hatching. Scale bar represents $50 \mu \mathrm{m}$. (TIF $31728 \mathrm{~kb}$ )

Additional file 2: Table S1. Detailed information for each sample used. Sample ID, Barcodes, Read recovered etc. (CSV $12 \mathrm{~kb}$ )

Additional file 3: Table S2. Full genotypes for mapping family (CSV $301 \mathrm{~kb}$ )

Additional file 4: Table S3. Consensus genetic map (positions and RAD locus sequences). (CSV $235 \mathrm{~kb}$ )

Additional file 5: Figure S2. Female only map of all SNP markers. The positions on the left side of chromosomes are the distance in centi Morgan (cM), Detailed data is provided in Table S2. (PDF $849 \mathrm{~kb}$ )

Additional file 6: Table S4. Female only genetic map (positions and RAD locus sequences). (CSV $122 \mathrm{~kb}$ )

Additional file 7: Figure S3. Male only map of all SNP markers. The positions on the left side of chromosomes are the distance in centi Morgan (CM), Detailed data is provided in Table S2. (PDF $850 \mathrm{~kb}$ )

Additional file 8: Table S5. Male only genetic map (positions and RAD locus sequences). (CSV $130 \mathrm{~kb}$ )

Additional file 9: Table S6. Primer and sequence information for allele-specific SNP assays. (CSV 597 bytes)

Additional file 10: Figure S4. Gel images of SNP assays for Sphl8298 and Sphl6331. (PDF $209 \mathrm{~kb})$

Additional file 11: Table S7. Synteny comparisons. Location of each marker retrieved in tested genomes. For each marker, the chromosome ID, start and end location of the best BLASTn hit is reported. (CSV $138 \mathrm{~kb}$ )

\section{Abbreviations}

CM, centiMorgans; ddRAD, double digest Restriction-site Associated DNA; FCR, feed conversion ratio; LOD, logarithm of the odds; NGS, next generation sequencing; NIWA, New Zealand National Institute of Water and Atmospheric Research; PIT, passive integrated transponders; RAD, Restriction-site Associated $D N A ; R E$, restriction enzyme; SNP, Single Nucleotide Polymorphism.

\section{Acknowledgements}

We are grateful to Jacquie Ireland (Institute of Aquaculture, School of Natural Sciences, University of Stirling) for providing technical assistance. We also are grateful for the support and assistance of the staff and students at NIWA, Bream Bay Aquaculture Park and GenomNZ (AgResearch). Funding for this study was received through the NIWA Research Aquaculture Programmes 1 and $3(2014 / 15 \mathrm{SCl})$. The authors also acknowledge the support from the MASTS pooling initiative (The Marine Alliance for Science and Technology for Scotland) in the completion of this study. MASTS is funded by the Scottish Funding Council (grant reference HR09011) and contributing institutions.

\section{Authors' contributions}

JKB extracted and purified the genomic DNA, performed the restriction enzyme digests, assisted in preparation of the libraries and genotyping of ddRAD alleles, generated the genetic linkage maps, performed the association analysis, verified the allele-specific PCR assays, analysed and interpreted the data and drafted the initial manuscript. JBT conceived and designed the molecular genomics component of the study, played the lead role in preparing the libraries and genotyping of ddRAD alleles, designed the allele-specific PCR assays and was involved in data interpretation. MB performed extra bioinformatics analyses, advised on interpretation of the data and on bioinformatics. SW performed the comparative genomics and advised on bioinformatics. CP advised on bioinformatics and interpretation of data. ANS conducted the animal work and histology at NIWA. JES conceived the study, supervised the animal work and histology at NIWA and provided the fin clips. DJP conceived and designed the study, advised on interpretation of the data. All authors read, edited and approved the manuscript.

\section{Competing interests}

The authors declare that they have no competing interests. The study sponsor, NIWA, is a Crown Research Institute of New Zealand that conducts commercial and non-commercial research across a broad range of disciplines in the environmental sciences.

\section{Author details}

'Institute of Aquaculture, School of Natural Sciences, University of Stirling, Stirling FK9 4LA, Scotland, UK. ${ }^{2}$ The Roslin Institute, Royal (Dick) School of Veterinary Studies, University of Edinburgh, Easter Bush, Midlothian EH25 9RG, Scotland, UK. ${ }^{3}$ New Zealand National Institute of Water and Atmospheric Research, Bream Bay Aquaculture Park, Station Road, Ruakaka 0151, New Zealand. ${ }^{4}$ Cawthron Institute, 98 Halifax Street East, Nelson 7010, New Zealand.

Received: 22 December 2015 Accepted: 25 May 2016

Published online: 10 June 2016

\section{References}

1. FishBase, Froese R, Pauly D, Editors. http://www.fishbase.org, Accessed 01 July 2015.

2. Barreiros JP, Machado L, Hostim-Silva M, Sazima I, Heemstra PC. First record of Polyprion oxygeneios (Perciformes: Polyprionidae) for the southwest Atlantic and a northernmost range extension. J Fish Biol. 2004;64:1439-41.

3. Francis MP, Mulligan KP, Davies NM, Beentjes MP. Age and growth estimates for New Zealand hāpuku, Polyprion oxygeneios. Fish B-NOAA. 1999;97:227-42.

4. Wakefield CB, Newman SJ, Molony BW. Age-based demography and reproduction of hāpuku, Polyprion oxygeneios, from the south coast of Western Australia: implications for management. ICES J Mar Sci. 2010;67:1164-74.

5. Papandroulakis N, Suquet M, Spedicato MT, Machias A, Fauvel C, Divanach P. Feeding rates, growth performance and gametogenesis of wreckfish (Polyprion americanus) kept in captivity. Aquacult Int. 2004;12:395-407.

6. Khan JR, Pether S, Bruce M, Walker SP, Herbert NA. The effect of temperature and ration size on specific dynamic action and production performance in juvenile hāpuku (Polyprion oxygeneios). Aquaculture. 2015;437:67-74.

7. Land Based Aquaculture Assessment Framework. Modelled Species/Hãpuku http://www.lbaaf.co.nz/nz-aquaculture-species/modelled-species/hapuka/. Accessed 1 July 2015.

8. Symonds JE, Walker SP, Van de Ven I, Marchant A, Irvine GW, Pether S, et al. Developing broodstock resources for farmed marine fish. Proc N Z Soc Anim Prod. 2012;72:222-6

9. Anderson SA, Salinas I, Walker SP, Gublin Y, Pether S, Kohn YY, et al. Early development of New Zealand hāpuku Polyprion oxygeneios eggs and larvae. Jish Biol. 2012;80:555-71.

10. Kohn YY, Lokman P, Kilimnik A, Symonds JE. Sex identification in captive hāpuku (Polyprion oxygeneios) using ultrasound imagery and plasma levels of vitellogenin and sex steroids. Aquaculture. 2013;384-387:87-93.

11. Symonds JE, Walker SP, Pether S, Gublin Y, McQueen D, King A, et al. Developing yellowtail kingfish (Seriola lalandi) and hāpuku (Polyprion oxygeneios) for New Zealand aquaculture. N Z J Mar Fresh. 2014;48:371-84

12. Gamble T, Zarkower D. Sex determination. Curr Biol. 2012;22:R257-62.

13. Devlin RH, Nagahama Y. Sex determination and sex differentiation in fish: an overview of genetic, physiological, and environmental influences. Aquaculture. 2002;208:191-364

14. Penman DJ, Piferrer F. Fish gonadogenesis. Part I: Genetic and environmental mechanisms of sex determination. Rev Fish Sci. 2008;16:16-34. 
15. Peterson BK, Weber JN, Kay EH, Fisher HS, Hoekstra HE. Double digest RADseq: an inexpensive method for de novo SNP discovery and genotyping in model and non-model species. PLoS ONE. 2012;7:e37135.

16. Houston RD, Davey JW, Bishop SC, Lowe NR, Mota-Velasco JC, Hamilton A, et al. Characterisation of QTL-linked and genome-wide restriction site-associated DNA (RAD) markers in farmed Atlantic salmon. BMC Genomics. 2012;13:244.

17. Catchen JM, Amores A, Hohenlohe P, Cresko W, Postlethwait JH. Stacks: building and genotyping loci de novo from short-read sequences. G3. 2011;1:171-82

18. Rastas P, Paulin L, Hanski I, Lehtonen R, Auvinen P. Lep-MAP: fast and accurate linkage map construction for large SNP datasets. Bioinformatics. 2013;29:3128-34.

19. Kosambi DD. The estimation of map distances from recombination values. Ann Eugen. 1943;12:172-5.

20. Margarido GR, Souza AP, Garcia AA. OneMap: software for genetic mapping in outcrossing species. Hereditas. 2007;144:78-9.

21. Bekaert M. Genetic-Mapper. https://github.com/pseudogene/geneticmapper. Accessed 16 March 2016.

22. Gonzalez JR, Armengol L, Sole X, Guino E, Mercader JM, Estivill X, et al. SNPassoc: an $\mathrm{R}$ package to perform whole genome association studies. Bioinformatics. 2007;23:644-5.

23. Kwok S, Chang SY, Sninsky JJ, Wang A. A guide to the design and use of mismatched and degenerate primers. PCR Methods Appl. 1994;3:S39-47.

24. Altschul SF, Gish W, Miller W, Myers EW, Lipman DJ. Basic local alignment search tool. J Mol Biol. 1990;215:403-10.

25. Krzywinski M, Schein J, Birol I, Connors J, Gascoyne R, Horsman D, et al. Circos: an information aesthetic for comparative genomics. Genome Res. 2009;19:1639-45.

26. Palaiokostas C, Bekaert M, Taggart JB, Gharbi K, McAndrew BJ, Chatain B, et al. A new SNP-based vision of the genetics of sex determination in European sea bass (Dicentrarchus labrax). Genet Sel Evol. 2015;47:68.

27. Semagn K, Babu R, Hearne S, Olsen M. Single nucleotide polymorphism genotyping using Kompetitive Allele Specific PCR (KASP): overview of the technology and its application in crop improvement. Mol Breed. 2014;33:1-14.

28. Goddard M. Genomic selection: prediction of accuracy and maximisation of long term response. Genetica. 2009;136:245-57.

29. Vandeputte M, Dupont-Nivet M, Chavanne H, Chatain B. A polygenic hypothesis for sex determination in the European sea bass Dicentrarchus labrax. Genetics. 2007;176:1049-57.

30. Betancur-R R, Broughton RE, Wiley EO, Carpenter K, López JA, Li C, et al. The tree of life and a new classification of bony fishes. PLoS Curr. 2013;5: ecurrents.tol.53ba26640df0ccaee75bb165c8c26288

31. Garcia de la Serrana D, Mareco EA, Johnston IA. Systematic variation in the pattern of gene paralog retention between the teleost superorders Ostariophysi and Acanthopterygiienome. Biol Evol. 2014;6:981-7.

32. Dettai $A$, Lecointre $G$. Further support for the clades obtained by multiple molecular phylogenies in the acanthomorph bush. C R Biol. 2005;328:674-89.

\section{Submit your next manuscript to BioMed Central and we will help you at every step:}

- We accept pre-submission inquiries

- Our selector tool helps you to find the most relevant journal

- We provide round the clock customer support

- Convenient online submission

- Thorough peer review

- Inclusion in PubMed and all major indexing services

- Maximum visibility for your research

Submit your manuscript at www.biomedcentral.com/submit 